Kooperatives Politikmodell Integrierte Produktpolitik

\title{
Staatliches Handeln zwischen bleibenden Aufgaben und neuen Anforderungen
}

\author{
Die Politikmuster europäischen Regierens verändern sich. Entscheidungen wer- \\ den nicht mehr nur innerhalb des politischen Systems, sondern vermehrt von \\ Akteuren der Wirtschaft und Zivilgesellschaft (mit)gefällt. Integrierte Produkt- \\ politik steht beispielhaft für den Paradigmenwechsel von der Planungseuphorie \\ zur gesellschaftlichen Selbststeverung.
}

$\mathrm{D}$ Von Dirk Scheer und Frieder Rubik ie Erfahrungen in der Umweltpolitik verdeutlichen eine politische Akzentverschiebung. Hatten umweltpolitische Maßnahmen zu Beginn vor allem die Medien Luft, Wasser und Boden im Blick, um die dringlichsten Probleme nachsorgend zu lösen, so verpflichtet das Paradigma einer präventiven Umweltpolitik zu einem systematischen und ganzheitlichen Ansatz. Kern dieses Ansatzes ist die Idee einer lebenswegbezogenen Betrachtung. Der Ansatz Integrierte Produktpolitik (IPP) steht aus unserer Sicht beispielhaft für die Veränderungen, die die Politikmuster im Bereich der Umweltpolitik aber auch in zahlreichen anderen politischen Handlungsfeldern durchlaufen. Dieser Perspektivewechsel verdeutlichte allerdings zweierlei: die Komplexität der umweltpolitischen Problemlagen - und die Grenzen marktlicher wie staatlicher Problemlösungsfähigkeit.

\section{Die Grenzen des Marktes und des Stautes}

Märkte sind ein gutes Mittel, ökonomische Aktivitäten zu organisieren und zu strukturieren. Durch die „unsichtbare Hand“ (Adam Smith) kann es zu einer schöpferischen Zerstörung - wie dies einmal Schumpeter genannt hat - kommen. Märkte sind sensibel und bringen stetig neue Lösungen und Innovationen hervor. Allokationsprozesse erfolgen nach den Präferenzen der daran beteiligten Akteure. Märkte haben jedoch auch ihre Probleme - die Fachwelt spricht von einem Marktversagen. Beispiele sind die unzureichende Bereitstellung öffentlicher Güter, der Schutz der Umwelt oder der Umgang mit Informationsdefiziten.

Eine zentrale Rolle nimmt in diesem Zusammenhang oftmals der Staat ein, dessen Aufgabe es ist, für das Funktionieren von Märkten zu sorgen und öffentliche Leistungen zu erbringen. In der Vergangenheit wurde Politik zumeist als eine interventionistische, hierarchische Angelegenheit betrachtet, mit einem starken Staat, welcher der Zivilgesellschaft und den Märkten klare Rahmenbedingungen setzt. Allerdings sind auch dem Staat Probleme immanent, wie etwa Informationsdefizite, Anpassungskosten, Korruption, Bürokratie, Ineffizienz - hier sprechen Fachleute von einem Staatsversagen.

Die Grenzen von Markt und Staat beeinflussen auch den Bereich der Integrierten Produktpolitik. In diesem Politikfeld lässt sich ein strukturell bedingtes Staatsversagen ausmachen. Wichtige Gründe hierfür sind:

- Die regulatorisch ausgerichtete umweltpolitische Steuerungsstrategie, einzelfallbezogen Emissionen größerer stationärer Quellen zu begrenzen, stellt mittlerweile bereits aufgrund der Menge, der Entwicklungsdynamik sowie der Wechselwirkungen von Emissionen und Stoffen eine strukturelle Überforderung der Umweltpolitik dar.

- Systemisch bedingt können die Allokationskalküle und -entscheidungen der einzelwirtschaftlichen Akteure nicht vorhergesehen werden. Weder empirisch noch theoretisch kann der Staat sich das notwendige Systemwissen über mikroökonomische Entscheidungen und Verhaltensweisen beschaffen.

- Kapazitätsengpässe und die Fokussierung von Umweltpolitik setzen eine weitere Grenze: Staatlicherseits stehen nur begrenzte Kapazitäten bereit, die sich insbesondere auf prioritäre Umweltziele wie Klimaschutz konzentrieren.

Dennoch nimmt der Staat innerhalb einer IPP eine wichtige, weil tragende Rolle ein. Er ist in der
Lage, (umwelt-)politische Rahmenbedingungen vorzugeben. Um die selbstorganisierende Intention einer IPP gerecht zu werden, bedarf es der Umsetzung des im gesellschaftspolitischen Diskurs konstatierten Paradigmenwechsels: Die Rolle des Staates ist nicht auf seine command \& control-Funktion beschränkt, viel wichtiger werden seine push \& pull-Fähigkeiten zur Unterstiutzung von ökologischen Markttransformationen. Auffallend ist jedoch, dass Politik wie Wissenschaft der Entwicklung und Umsetzung einer systematischen Kooperationsstrategie in der Umweltpolitik bislang kaum Aufmerksamkeit geschenkt haben. Der kooperative Staat - ein produktpolitischer Papiertiger?

\section{Ansatzpunkt Kooperation}

Die kooperative, produktbezogene Umweltpolitik des Staates wird bislang vornehmlich auf der Ebene einzelner Politikinstrumente festgemacht. So ist den neuen Policy-Instrumenten zunächst gemeinsam, dass sie sich auf prozedurale Vorgaben beschränken. Sie legen bestimmte Verfahrensregeln fest, definieren jedoch keine substantiellen Ziele im Hinblick auf die Ergebnisse bestimmter Verfahren. Auffallend ist, dass jenseits der Instrumentenperspektive kaum ein strategisch ausgerichteter Diskurs über das Kooperationsprinzip in der Umweltpolitik existiert - dies gilt jedenfalls für den Bereich einer IPP. Die Erarbeitung von systematischen Kooperationsstrategien im Rahmen einer IPP steht denn auch erst am Anfang.

Die Entwicklung einer zukünftigen Kooperationsstrategie kann allerdings auf einige Ansatzpunkte und Erfahrungen rekurrieren, die als Bausteine für eine zukünftige gesellschaftliche Selbststeuerung im Rahmen einer IPP fungieren können. Dies möchten wir anhand eines stark vereinfachten Politikzyklus illustrieren.

1. Problemwahrnehmung - Sensibilisierung relevanter Akteure: Vorausschauende Umweltpolitik muss zukünftige Entwicklungen antizipieren, um Handlungsbedarf frühzeitig erkennen zu können. Für eine Kooperationsstrategie stellt sich die Herausforderung, Akteure aus Wirtschaft und Gesellschaft für produktinduzierte Umweltprobleme zu sensibilisieren und Handlungswillen bei den Akteuren zu generieren. Ein interessanter Ansatz ist das 1998 von der britischen Regierung eingeführte Market Transformation Programme, das zur Förderung energieeffizienter Endverbraucherprodukte mit Hilfe von Szenariotechniken quantitatives Datenmaterial generiert. Als Ergebnis des Sektor-Review-Prozesses werden öffentlich zugäng- 
liche Policy Briefs entwickelt, die Problemaufriss sowie Prioritäten und Aktionen entwerfen. Die Problemaufbereitung dient als Grundlage für Konsensbildungsprozesse mit allen relevanten Akteuren.

2. Politikformulierung - produktpolitisches Agenda Setting: Eine zentrale Herausforderung ist, Handlungsbedarf in konkrete Aktivitäten und Maßnahmen umzusetzen. Einige EU-Mitgliedsländer verfügen bereits über Erfahrung hinsichtlich kooperativer Politikformulierung. 1998 wurden von der dänischen Umweltbehörde für die Bereiche Textilien, Transport, Elektronik, Baumaterialien sowie Nahrungsmittel so genannte Product Panels eingeführt, um die Einbindung der Marktkräfte in die „Produktorientierte Umweltinitiative“ zu verstärken. Der Dialogprozess in den Product Panels mündet in Aktionsplänen, die eine Übersicht über alle wichtigen Aktivitäten in Bezug auf das betrachtete Feld und die Marktbedingungen enthalten.

Auf eine eher indirekte Weise kann auch die seit 1978 bestehende Jury Umweltzeichen genannt werden. Diese Jury ist pluralistisch zusammengesetzt und verfolgt damit einen kooperativen Ansatz. Aus kooperationsstrategischer Sicht ist weniger ihre direkte Hauptaufgabe bemerkenswert, nämlich die Festlegung von Vergabegrundlagen für den Blauen Engel, sondern ihre indirekte Wirkungen. Diskussionen in der Jury und während des gesamten Erarbeitungsprozesses bringen unterschiedliche Akteure zusammen und können als eine Art Frühwarnsystem für ökologische Probleme und Herausforderungen einer Produktgruppe aufgefasst werden, die auf einer mittelfristigen Zeitschiene bestehen.

In Großbritannien wurde 1999 das Advisory Committee on Consumer Products and the Environment (ACCPE) etabliert. Aufgabe des Expertengremiums aus Wirtschaft, Wissenschaft, Gesellschaft und Politik ist, die britische Regierung bezüglich der Reduktion von Umwelteinflüssen verursacht durch Produkte und Dienstleistungen zu unterstïtzen. Das ACCPE ist zum wichtigen Akteur des Agenda Settings für produktpolitische Fragen geworden. Das britische Umweltministerium verfolgt derzeit Planungen, die Institutionalisierung mit weiteren kooperativ ausgerichteten Expertengremien voranzutreiben. Im Gespräch sind derzeit die Schaffung einer so genannten Task Force on Production sowie ein Roundtable on Consumption.

3. Politikimplementierung - Kooperationsanreize durch umweltpolitische Instrumente: Zusammenarbeit zwischen gesellschaftlichen Akteuren kann auch indirekt durch die Politik initiiert werden - beispielsweise durch

\begin{tabular}{|c|c|c|c|}
\hline Politikphasen & Zielsetzung & Strategie & Leitprinzipien \\
\hline $\begin{array}{l}\text { Problem- } \\
\text { wahrnehmung }\end{array}$ & $\begin{array}{l}\text { - Sensibilisierung für } \\
\text { produktpolitischen } \\
\text { Handlungsbedarf }\end{array}$ & $\begin{array}{l}\text { - Konsensorientierter } \\
\text { Dialog mit Akteuren }\end{array}$ & $\begin{array}{l}\text { - Konsensbildung über } \\
\text { (produktgruppenspezifische) } \\
\text { Handlungsnotwendigkeit } \\
\text { - Wissenschaftlich fundierter } \\
\text { Problemaufriss }\end{array}$ \\
\hline Politikformulierung & $\begin{array}{l}\text { - Kooperative Verständigung } \\
\text { Über Prioriäten und Vorgehen }\end{array}$ & $\begin{array}{l}\text { - Stärkung des } \\
\text { produktpolitischen } \\
\text { Agenda Setting }\end{array}$ & $\begin{array}{l}\text { - Institutionalisierung/Bündelung } \\
\text { produktpolitischer Expertise } \\
\text { - Integration in politisch-gesellschaft- } \\
\text { lich Diskurse (z.B. Klimapolitik, Nach- } \\
\text { haltigkeit, Corporate Responsibility) } \\
\text { - Produktpolitische Prioritätensetzung } \\
\text { und Zieldefinition (Roadmap IPP) }\end{array}$ \\
\hline $\begin{array}{l}\text { Politik- } \\
\text { implementierung }\end{array}$ & $\begin{array}{l}\text { - Verbesserung der Umwelt- } \\
\text { bzw. Nachhaltigkeitseffekte } \\
\text { von Produkten }\end{array}$ & $\begin{array}{l}\text { - Kooperationsanreize } \\
\text { durch umweltpolitische } \\
\text { Instrumente }\end{array}$ & $\begin{array}{l}\text { - Produktverantwortung } \\
\text { - Life-cycle thinking } \\
\text { - Product chain innovation }\end{array}$ \\
\hline
\end{tabular}

Kooperationsanreize qua Integration in politische Instrumentarien. Ein Blick auf umweltpolitische EU-Richtlinien, die den Lebensweggedanken berücksichtigen, kann dies illustrieren. So sind nach der so genannten WEEE-Richtlinie die Hersteller verpflichtet, individuell oder kollektiv Systeme für die Verwertung von Elektro- und Elektronik-Altgeräte einzurichten. Ähnlich verhält es sich bei der so genannten Alt-Auto-Richtlinie, die vorschreibt, dass Normen zur Recyclingfähigkeit von Neufahrzeugen zu schaffen und für die Typgenehmigung von Neufahrzeugen vorzuschreiben sind sowie Kennzeichnungsstandards für wieder verwertbare Bauteile eingeführt werden. Die stärkere Berücksichtigung des Lebenswegsgedankens in der Gesetzgebung, der eine enge Zusammenarbeit zwischen den Akteuren des gesamten Lebensweges initiiert, kann zu einem zentralen Baustein einer Kooperationsstrategie werden.

\section{Grenzen von Kooperationen}

Kooperationen sind allerdings nicht immer geeignet, adäquate Antworten auf umweltpolitische Probleme zu finden. Gerade im Produktbereich finden sie ihre Grenzen dort, wo es um Gefahrenabwehr und Risikovorsorge geht. In diesen Fällen sind Interventionen seitens des Staates notwendig. Beispiele aus jüngster Zeit sind vor allem im Bereich der Gesundheitsvorsorge zu nennen, wie etwa die BSE-Krise. Intervention und ordnungsrechtliche Regulierung wird sich jedoch auf Hot Spots begrenzen.

Zusammenfassend sehen wir einen dualen Weg. Eine stärkere staatliche Intervention in Fällen von Gefahren und Risiken sowie ein kooperativer Ansatz im Falle von Herausforderungen und Problemen, die mittel- bis langfristig sind und keine ge-
Quelle: eigene Darstellung

sellschaftliche Hot Spots darstellen. Strukturelle Risiken mittel- und langfristiger Art können nur fallweise mit diesem Ansatz begegnet werden. Hierbei ist insbesondere ein proaktiver Ansatz zu verfolgen, der im Falle einer IPP ein Andocken an die aktuelle Innovationsdebatte erfordert und über Kooperationen verlässliche Leitplanken schafft. Eine Kultur der Kooperation wird eine Kultur nachhaltiger Innovation erleichtern. Dabei wird sich die bisherige Fokussierung auf einzelne Instrumente und Stoffe zugunsten einer mehrdimensionalen kooperativen Herangehensweise verschieben. Konzeptionell stellt dies auch eine Herausforderung für die Wissenschaft dar, die der Frage nach einer systematischen Gestaltung von Kooperationen im Bereich der Integrierten Produktpolitik bisher wenig Aufmerksamkeit gewidmet hat.

Anmerkung

(1) Siehe auch Rubik, F./ Keil, M.: Kooperative Ansätze im Rahmen einer Integrierten Produktpolitik. Broschüre des Ministeriums für Umwelt und Verkehr, Stuttgart, im Erscheinen.

Dr. Frieder Rubik ist Leiter des Forschungsfelds Öko logische Produktpolitik des Instituts für ökologische Wirtschaftsforschung (IÖW). Dirk Scheer ist im selben Forschungsfeld wissenschaftlicher Mitarbeiter. Kontakt: IÖW (Büro Heidelberg), Bergstr. 7, 69120 Heidelberg. Tel. 06221-649163, Fax 0622127060, E-Mail: dirk.scheer@heidelberg.ioew.de, frieder.rubik@ioew.de

\section{Die Autoren}


(c) 20I0 Authors; licensee IÖW and oekom verlag. This is an article distributed under the terms of the Creative Commons Attribution Non-Commercial No Derivates License (http://creativecommons.org/licenses/by-nc-nd/3.o/), which permits unrestricted use, distribution, and reproduction in any medium, provided the original work is properly cited. 